\title{
Estudo Prospectivo de Documentos de Patentes Relacionados à Produção de Bebidas Alcoólicas Fermentadas de Frutas
}

\author{
Prospective Study of Patent Documents Related to the Production of \\ Alcoholic Beverages Fermented Fruit
}

\author{
Renata Torres dos Santos e Santos ${ }^{1}$ \\ Clívia Danubia Pinho da Costa Castro ${ }^{2}$ \\ Ana Cecilia Poloni Rybka ${ }^{2}$ \\ Aline Camarão Telles Biasoto ${ }^{2}$ \\ Flávio Luiz Honorato da Silva ${ }^{1}$ \\ ${ }^{1}$ Universidade Federal da Paraíba, João Pessoa, PB, Brasil \\ ${ }^{2}$ Empresa Brasileira de Pesquisa Agropecuária, Petrolina, PE, Brasil
}

\begin{abstract}
Resumo
Com o avanço tecnológico, a produção mundial de frutas tem apresentado crescimento contínuo, o que aponta para certo beneficiamento na elaboração de bebidas alcoólicas. Este trabalho faz uma prospecção tecnológica com o objetivo de mapear documentos de patentes que se referem à elaboração de bebidas alcoólicas fermentadas de frutas. Para tanto, serão utilizadas as bases de dados on-line EPO e INPI, combinando os descritores fermented* fruit* e wine* com os códigos da Classificação Internacional C12G3/00 e C12G3/02 e as palavras-chave "fermentado de fruta", "fermentado alcoólico" e "vinho de fruta". A partir dos dados obtidos, constatou-se 344 patentes no período de 2003 e 2018. A China destaca-se por deter aproximadamente 95\% dos documentos selecionados e por apresentar a maior representabilidade nos principais inventores e depositantes (instituições acadêmicas e indústrias). O mirtilo, a amora e o kiwi foram as frutas mais representativas, seguidas da ameixa, pera e manga. Diante do exposto, verificouse uma tendência no crescimento de depósitos patentários na área de bebidas alcoólicas. No entanto, embora muitas frutas sejam conhecidas no preparo desses derivados, ainda há variedades pouco exploradas nesse campo.
\end{abstract}

Palavras-chave: Frutos. Fermentado Alcóolico. Patente.

\begin{abstract}
With the technological advance, the world fruit production presents a continuous growth, citing its beneficiation in the elaboration of alcoholic beverages. This technological prospection aimed at the mapping of patent documents covering the production of fermented alcoholic beverages using the online databases EPO and INPI, combining the descriptors fermented * fruit * and wine * with the international classification codes C12G3/00 and C12G3/02, and the keywords fermented fruit, alcoholic fermented and fruit wine. A total of 344 patents were registered in the period 2003 and 2018. China was noted for holding approximately 95\% of the documents selected; as well as for presenting the greatest representation in the main inventors and depositors (academic institutions and industries). Blueberry, blackberry and kiwi were the most representative fruits, followed by plum, pear and mango. Given the above, there was a tendency to grow patented deposits in the area of alcoholic beverages; and although many fruits are known in the preparation of these derivatives, there are still little explored varieties in this field.
\end{abstract}

Keywords: Fruits. Fermented alcoholic beverage. Patent.

Área Tecnológica: Propriedade Intelectual, Inovação Tecnológica, Ciência dos Alimentos. 


\section{Introdução}

A produção mundial de frutas tem apresentado um crescimento contínuo devido ao grande avanço tecnológico que tem ocorrido no mundo. Nesse cenário, há uma abundante variedade de frutas cultivadas com potencial para serem utilizadas pela indústria de alimentos. A China, a Índia e o Brasil, juntos, respondem por quase metade dessa produção mundial e têm suas produções destinadas principalmente aos seus mercados internos (SEBRAE, 2015).

No Brasil, entre sua diversidade de espécies cultivadas, destaca-se a produção de frutas de clima temperado e subtropical, com colheitas significativas para a laranja, banana, abacaxi, melancia, coco, uva, mamão, maçã, limão e tangerina (SEAB, 2017).

Entre as diversas formas de consumo, cita-se o beneficiamento na elaboração de bebidas alcoólicas, por exemplo: licores, fermentados, destilados e outros. De acordo com a legislação brasileira, o fermentado de fruta se caracteriza como uma bebida com graduação alcoólica de 4 a $14 \% \mathrm{v} / \mathrm{v}$, a $20^{\circ} \mathrm{C}$, e um percentual de açúcar máximo de $3 \mathrm{~g} / \mathrm{L}$, se classificado como seco, ou mínimo de $3 \mathrm{~g} / \mathrm{L}$, se considerado doce ou suave, e deve ser obtido a partir da fermentação do suco de uma única fruta, podendo ser utilizados a água e o açúcar como ingredientes permitidos; o fermentado de fruta licoroso, doce ou seco, com graduação alcoólica de 14 a 18\% v/v, a $20^{\circ} \mathrm{C}$, adicionado ou não de álcool etílico potável de origem agrícola, caramelo e sacarose; e o fermentado de fruta composto, com graduação alcoólica de 15 a $20 \% \mathrm{v} / \mathrm{v}$, a $20^{\circ} \mathrm{C}$, obtido pela adição ao fermentado de fruta, de macerados ou extratos de plantas amargas ou aromáticas, adicionado ou não de álcool etílico potável de origem agrícola, caramelo e sacarose (BRASIL, 2008; 2012).

De acordo com Rosa, Cosenza e Leão (2006), uma forte tendência do setor de bebidas é a de diversificação de sabores e tipos de bebidas. A diversidade das frutas existentes com propriedades adequadas para o processamento desses produtos demonstra que esse é um mercado que tem potencial para crescer, além de representar uma forma alternativa para contornar os problemas das perdas pós-colheita devido à perecividade dos frutos.

Embora muitas frutas sejam conhecidas no preparo desses derivados, ainda há algumas variedades pouco exploradas nesse campo. Dessa maneira, o presente estudo foi desenvolvido com o intuito de realizar um mapeamento prospectivo, a nível nacional e internacional, da produção de bebidas alcoólicas fermentadas de frutas.

\section{Metodologia}

O estudo prospectivo foi realizado em maio e junho de 2018, e as buscas e o levantamento dos documentos de patentes foram realizados por meio do banco de dados on-line do Instituto Nacional da Propriedade Intelectual (INPI) e do European Patent Office (EPO) (Espacenet $\left.{ }^{\circledR}\right)$.

A pesquisa buscou realizar o maior levantamento possível do número de documentos de patentes relacionadas ao tema de interesse. Dessa forma, para que se tornar uma pesquisa representativa e fiel, foi necessário estabelecer uma estratégia de combinações de palavras-chave, inseridas no campo "Título ou Resumo", com os códigos da Classificação Internacional de Patentes (CIP), inseridos no campo "IPC", pertencentes à Seção C - Química, por meio da busca avançada. 
As palavras-chave utilizadas no INPI foram "fermentado de fruta", "fermentado alcoólico" $e$ "vinho de fruta". E os descritores utilizados na EPO foram fermented* fruit* (fermentado de fruta) e wine* (vinho), combinados com os códigos IPC C12G3/00 e C12G3/02. As Tabelas 1, 2 e 3 mostram o escopo utilizado para o mapeamento da pesquisa dos documentos de patentes e a classificação de cada código utilizado na busca, respectivamente.

Tabela 1 - Resultados da busca em banco de dados de patente do INPI

\begin{tabular}{cccc} 
Palavras-chave & \multicolumn{2}{c}{ Campus da busca } & Resultado \\
\cline { 2 - 3 } fermentado de fruta & Título & Resumo & 0 \\
fermentado de fruta & $\mathrm{x}$ & $\mathrm{x}$ & 8 \\
vinho de fruta & & $\mathrm{x}$ & 0 \\
vinho de fruta & $\mathrm{x}$ & & 7 \\
fermentado alcoólico & & $\mathrm{x}$ & 0 \\
fermentado alcoólico & $\mathrm{x}$ & 5 \\
\hline
\end{tabular}

Fonte: Elaborada pelos autores deste artigo a partir da base de dados INPI (2018)

Tabela 2 - Códigos de IPC utilizados para o mapeamento da prospecção tecnológica

$\begin{array}{cc}\text { Código IPC } & \text { CLAssificaÇÃo } \\ \text { C12G3/00 } & \text { Preparação de outras bebidas alcoólicas. } \\ \text { C12G3/02 } & \text { Por fermentação direta. }\end{array}$

Fonte: EPO (2018)

Tabela 3 - Descritores Espacenet versus Códigos de Classificação Internacional

\begin{tabular}{|c|c|c|c|}
\hline \multirow[t]{2}{*}{ Título ou RESUmo } & \multicolumn{2}{|c|}{ Código IPC } & \multirow[t]{2}{*}{ Resultado } \\
\hline & $\mathrm{C} 12 \mathrm{G} 3 / 00$ & $\mathrm{C} 12 \mathrm{G} 3 / 02$ & \\
\hline fermented drink and fruit & & & 308 \\
\hline fruit wine & & & 8821 \\
\hline fruit & $\mathrm{x}$ & & 297 \\
\hline fruit wine & $\mathrm{x}$ & & 143 \\
\hline fruit wine & & $\mathrm{x}$ & 3013 \\
\hline fruit wine & $\mathrm{x}$ & $\mathrm{x}$ & 3127 \\
\hline fermented drink and fruit & $\mathrm{x}$ & $\mathrm{x}$ & 55 \\
\hline fruit drink & $\mathrm{x}$ & $\mathrm{x}$ & 264 \\
\hline fermented drink & $\mathrm{x}$ & $\mathrm{x}$ & 913 \\
\hline fermented fruit & & $\mathrm{x}$ & 889 \\
\hline fermented fruit and wine & $\mathrm{x}$ & $\mathrm{x}$ & 776 \\
\hline fermented* fruit* and wine* & $\mathbf{x}$ & $\mathbf{x}$ & 879 \\
\hline
\end{tabular}

Fonte: Elaborada pelos autores deste artigo a partir da base de dados EPO (2018) 
No INPI, o resultado escolhido foi aquele da busca em que as palavras-chaves "fermentado de fruta" $e$ "vinho de fruta" foram utilizadas no campo do resumo, para a primeira palavra-chave foram obtidos oito documentos e, para a segunda, sete. Desses documentos, apenas cinco foram selecionados para o mapeamento do estudo.

$\mathrm{Na}$ EPO, o resultado escolhido para o estudo foi a busca que apresentou 879 documentos associando as palavras-chave truncadas fermented* fruit* e wine* com os códigos da CIP C12G3/00 e C12G3/02; interligados pelo operador booleano "and". Desse resultado, apenas 500 documentos foram disponibilizados para consulta; os quais foram compactados e exportados para o software Comma-separated values (CSVed), editor de arquivos que manipula e separa os arquivos com qualquer tipo de separador. A partir desse software, os dados foram exportados para a ferramenta de planilha da Microsoft Office, Excel 2013, com o propósito de realizar a seleção e o tratamento das informações. Ao final desse procedimento, 342 documentos de patentes depositadas foram selecionados para o mapeamento do estudo.

\section{Resultados e Discussão}

A evolução anual depósitos de patentes relacionadas às bebidas fermentadas, que têm em sua composição alguma variedade de fruta, entre 2000 a 2018 nas bases de dados INPI e EPO, encontra-se exibida na Figura 1.

Figura 1 - Temporalidade dos registros de patentes depositadas no Espacenet entre 2000-2018

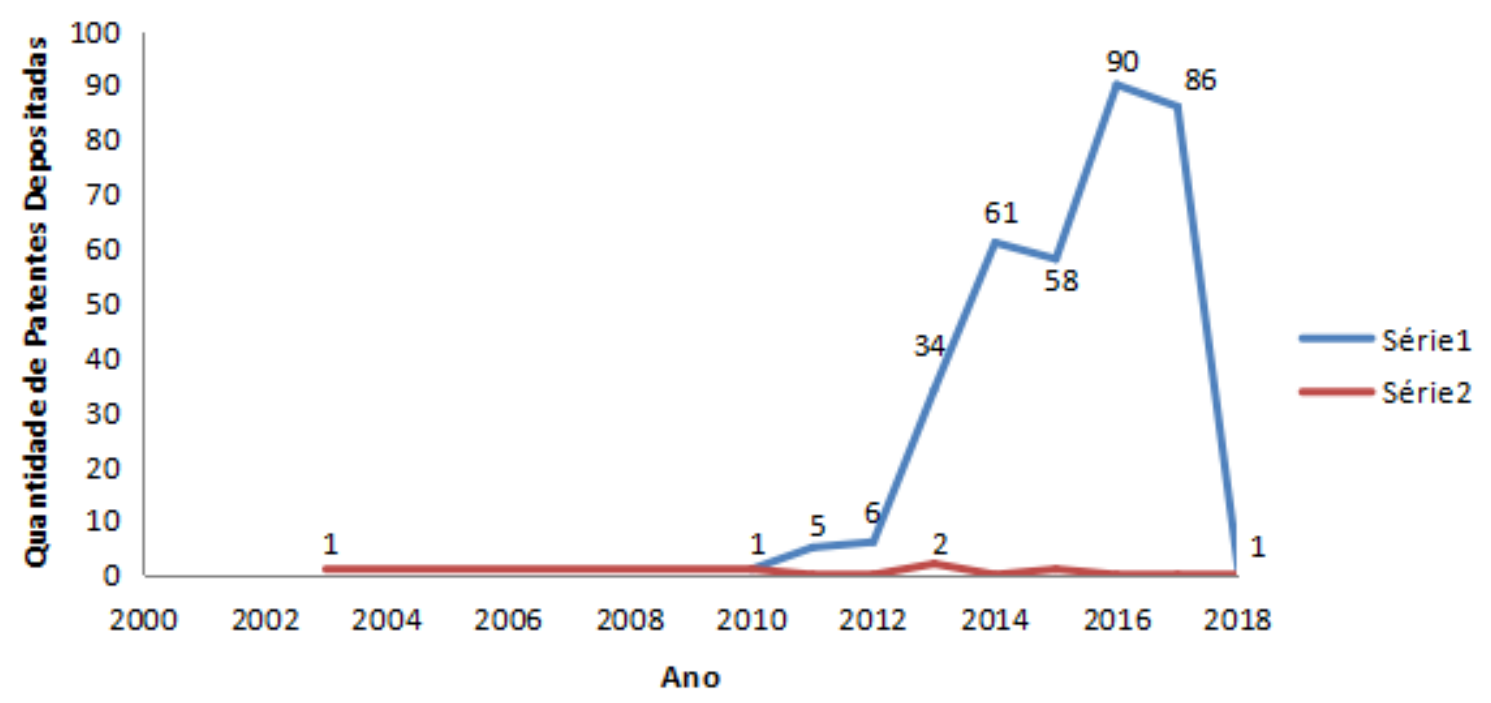

Série 1: EPO. Série 2: INPI.

Fonte: Elaborada pelos autores deste artigo a partir das bases de dados INPI (2018) e EPO (2018)

O resultado da busca revelou que a primeira patente deste estudo, registrado no INPI (C1 9703727-3 F1), ocorreu em 2003 sob a titularidade do Brasil e foi depositada pelo inventor independente Padre José Eduardo Pereira, no Rio de Janeiro, em que ele descreve o processo para a fabricação de vinho licoroso, seco, suave, espumante, vinagre, geleia, licor e extratos derivados do jamelão. Em seguida, em 2010, ainda no INPI, a patente PI 1005479-0 A2 sob a titularidade da Universidade Federal do Espírito Santo descreve a bebida alcoólica fermentada 
de café e o processo para a sua produção; e, na EPO, a patente UA94878 (C2) sob a titularidade da Ucrânia foi depositada pelo Instituto de Horticultura da Academia Ucraniana de Ciências Agrárias e descreve o processo de elaboração de um vinho de frutas a partir do suco fermentado de ameixa com o suco fortificado de cereja e utilização do açúcar granulado.

Por conseguinte, em 2011, foram depositadas cinco patentes na área em estudo que descrevem: processo de produção de um vinho de fruta fermentado a partir da cereja (CN103184122 (A)); processo de produção de vinho de frutas com cogumelos e abacaxi (CN103184121 (A)); processo de invenção do vinho de fruta fermentado com amora (CN103103048 (A)); método para a fabricação de vinho de kiwi (KR101246093 (B1)); e método para fabricar vinho tradicional usando frutas de ginseng (KR20130021619 (A)).

No ano seguinte, em 2012, foram depositadas seis patentes, as quais descrevem: método de produção de um vinho de frutas utilizando mel (KR20140071672 (A)); método de produção do vinho de nêspera (CN103789127 (A)); método de preparação do vinho de canistel (CN103571680 (A)); método para fermentar o vinho de amora (CN103361216 (A)); tecnologia de fabricação de vinho de kiwi (CN103173311 (A)); e preparo de vinho de frutus rhodomyrti (CN103045430 (A)).

Em 2013, no INPI, houve o depósito das patentes BR 1020130170348 A2 e BR 102013 008434-4 A2 pela Universidade Federal de Goiás e pela Universidade Estadual de Ponta Grossa, as quais descrevem, respectivamente, "Processo para elaboração de um fermentado alcoólico de polpa de baru (Dipteryx Alata Vog.)" e "Desenvolvimento de fermentado frisante de maçã, usando tecnologia europeia de processamento e atributos de aceitação do consumidor brasileiro". Ainda no INPI, ressalta-se a patente BR 102015031663-1 A2, depositada em 2015, que descreve "Bebida fermentada de açaí e respectivo processo de fabricação e engarrafamento", sob a titularidade do inventor independente Helder Juber Ehm Maia.

A partir do que foi exposto, observa-se o depósito contínuo de patentes na área. Sendo possível verificar que a maior ocorrência de documentos depositados ocorreu a partir do ano 2013, com 34 patentes na EPO e duas patentes no INPI, com ápice para os anos de 2016 e 2017, com 90 e 86 documentos de patentes, respectivamente, na EPO. Esse sensível aumento ocorreu, possivelmente, por incentivos e melhorias na tecnologia das indústrias no todo e por causa de investimentos em ciência, pesquisa e tecnologia.

Em 2018 ocorreu um decréscimo na quantidade de patentes que pode estar associado ao sigilo de 18 meses dos documentos antes de eles serem reveladas nas bases de patentes. Assim, houve apenas um documento de patente em 2018, depositada pela Universidade Estadual de Ifugão nas Filipinas; a patente (PH22016001018 (U1)) descreve o processo de produção de vinho a partir de vegetais e de frutos do tomate e do mamão.

Como detentores de documentos de patentes de bebidas fermentadas com frutas, verifica-se a China, a República da Coreia, as Filipinas, o Brasil, a Espanha, a Ucrânia e a Organização Mundial da Propriedade Intelectual (OMPI)), conforme apresentado na Figura 2. Desses detentores, ressalta-se a China que detém 93,7\% dos documentos de patentes, com 325 depósitos. Os demais países representam $6,3 \%$ da pesquisa e apresentaram quantidade total de depósitos igual a 22 patentes. 
Figura 2 - Quantitativo de patentes referentes ao fermentado de fruta por país

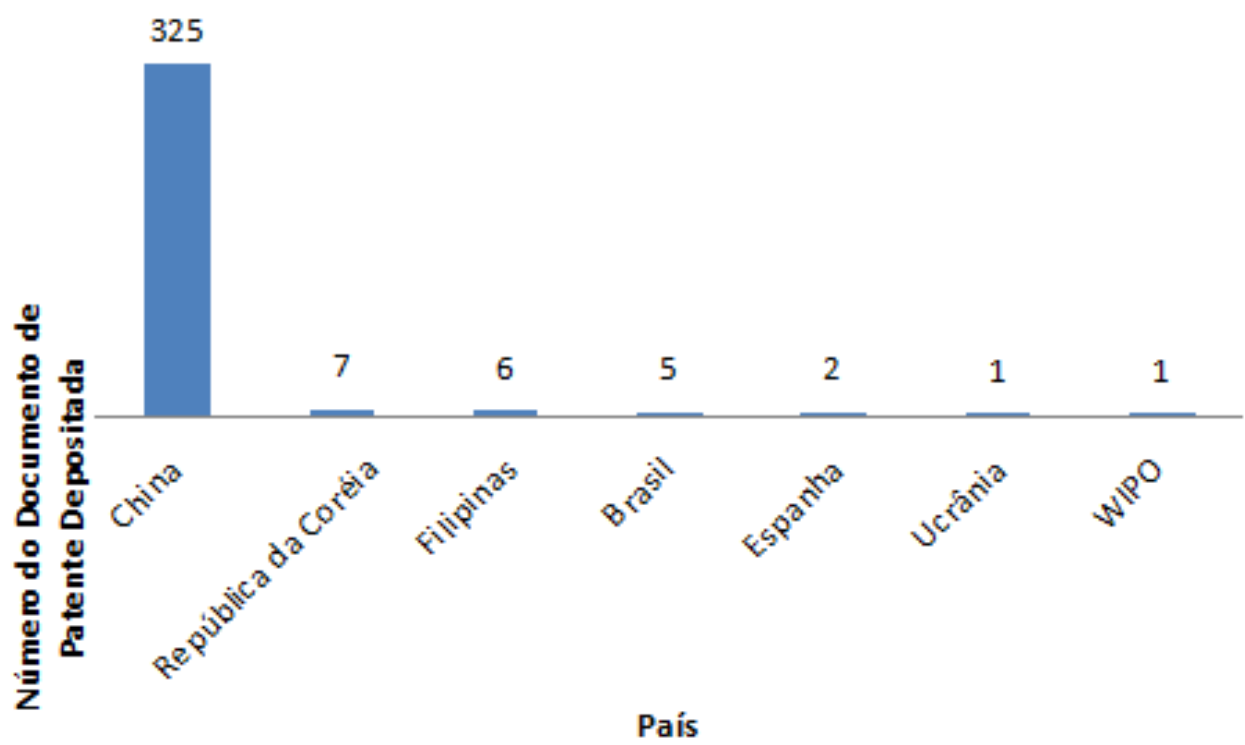

Fonte: Elaborada pelos autores deste artigo a partir das bases de dados INPI (1018) e EPO (2018)

A China que é atualmente uma das potências mais importantes para a economia do mundo também ocupa o primeiro lugar mundial na solicitação do número de patentes (WIPO, 2017) e o primeiro lugar no ranking mundial na produção de frutas, destacando-se com as produções de melancia, maçã, manga, melão, tangerina, pera, pêssego, nectarina e ameixa (SEAB, 2017). Tal informação permite deduzir que o país aplica investimentos na agricultura. $\mathrm{O}$ quantitativo elevado de documentos de patentes depositado pelo país é voltado para a indústria de alimentos, mostrando que investimentos e recursos estão sendo alocados para o desenvolvimento dessas patentes.

O Brasil ocupa o décimo primeiro lugar em solicitação de depósito de patentes (WIPO, 2017), embora seja reconhecido pela grande variedade de frutas produzidas em todo o país, atualmente está ocupando o terceiro lugar em produção mundial de frutas (SEBRAE, 2016) e aparece como detentor de apenas cinco documentos de patentes na plataforma INPI. Dessa forma, percebe-se que os incentivos à propriedade intelectual no país ainda são pequenos, uma vez que não há ampla divulgação sobre a importância da criação de patentes, esclarecimentos e sobre informações de que proteger uma invenção é contribuir para o desenvolvimento de um país. Por meio das patentes, as nações podem garantir a transferência de tecnologias, obter lucros, conquistar novos mercados e divisas. O depósito de patentes dinamiza o potencial tecnológico e reafirma a identidade de um povo, fortalecendo, inclusive, sua soberania e economia.

No entanto, ao analisar os dados dos últimos rankings publicados pela World Intellectual Property Indicators, pode-se observar que o Brasil tem apresentado um pequeno crescimento na solicitação de documentos de patentes ao decorrer dos anos; por exemplo, analisando os anos de 2010, 2012, 2014 e 2016, percebe-se que foram depositados, respectivamente, 22.686, 30.116, 30.342 e 28.010 documentos de patentes (WIPO, 2011; 2013; 2015; 2017). Esses dados demonstram que a propriedade intelectual no país vem se propagando a cada ano, o que pode vir a reverter, no futuro, os resultados das pesquisas realizadas nas bases de dados.

Adicionalmente, ao analisar a titularidade por setores da sociedade (Figura 3), observa-se que a maior parte dos documentos de patentes foi depositada pelas indústrias, seguidas pelas 
instituições de ensino e pesquisa (representadas pelas universidades $e$ institutos acadêmicos) $e$ pelos inventores independentes, detendo, respectivamente, $41 \%$, 33\% e $26 \%$ dos documentos depositados.

Figura 3 - Percentual de patentes depositadas por setores da sociedade

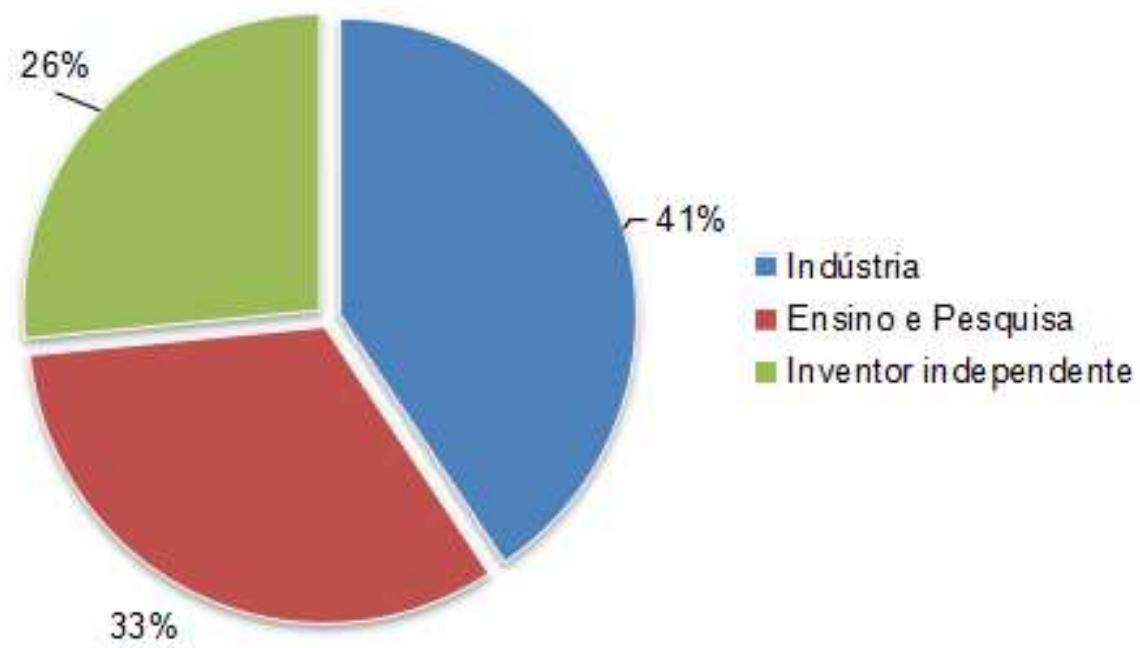

Fonte: Elaborada pelos autores deste artigo a partir das bases de dados INPI (2018) e EPO (2018)

Nesse contexto, os inventores que se destacam pela quantidade relevante de documentos de patentes depositados estão apresentados na Figura 4. Entre eles, Huang Ye, Feng Tao, Lin Guoyou e Wang Hui se destacaram com 22, 14 e 12 depósitos, respectivamente. Esses depósitos com os demais pertencem à China, país detentor principal das patentes selecionadas neste estudo.

Por conseguinte, entre os inventores apresentados, percebe-se que, na grande maioria, suas patentes foram depositadas por instituições privadas, principalmente pelas indústrias que detêm quantidade relevante das patentes selecionadas. Isso comprova que as patentes depositadas são financiadas pelo setor industrial com o intuito de gerar rentabilidade pela inovação e/ou pelo desenvolvimento de uma nova patente que potencialize os lucros e a comercialização de seus produtos.

Figura 4 - Inventores com maior incidência de patentes depositadas

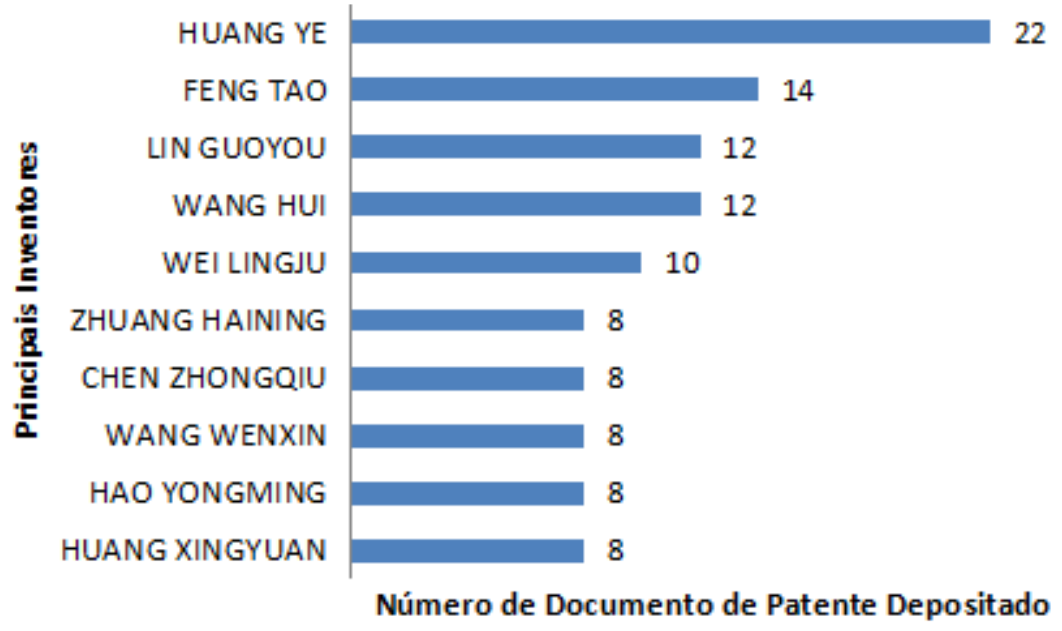

Fonte: Elaborada pelos autores deste artigo a partir das bases de dados INPI (2018) e EPO (2018) 
Os países com quantidade relevante de depósitos estão apresentados na Figura 5. Da relação, as instituições acadêmicas de ensino e pesquisa Shanghai Inst Technology e Liuzhou Lianhai Science \& Technology Co Ltd se destacam por deterem oito e sete documentos de patentes, respectivamente. Na sequência, a empresa Anhui Chenyaohu Rice Wine Co Ltd vem com cinco documentos de patentes. Nota-se, ainda, que todos esses depósitos ocorreram entre 2014 e 2017, período de ápice de depósitos observado neste estudo.

O Instituto de Tecnologia de Xangai, na China, é uma instituição de ensino superior em tempo integral, constituído por um Centro de Educação Moderna, dois departamentos e 11 escolas, que tem a biotecnologia e a engenharia de processamento de alimentos como alguns de seus campos de estudo. A Liuzhou Lianhai Science \& Technology Co Ltd é uma empresa de alta tecnologia especializada em transferência e em incubação de tecnologia internacional que tem como o seu principal negócio apresentar projetos de ciência e tecnologia de ponta, produzir novos produtos e, ao mesmo tempo, fornecer locais de incubação e serviços para atividades de inovação corporativas.

Figura 5 - Depositantes com maior incidência de documentos depositados

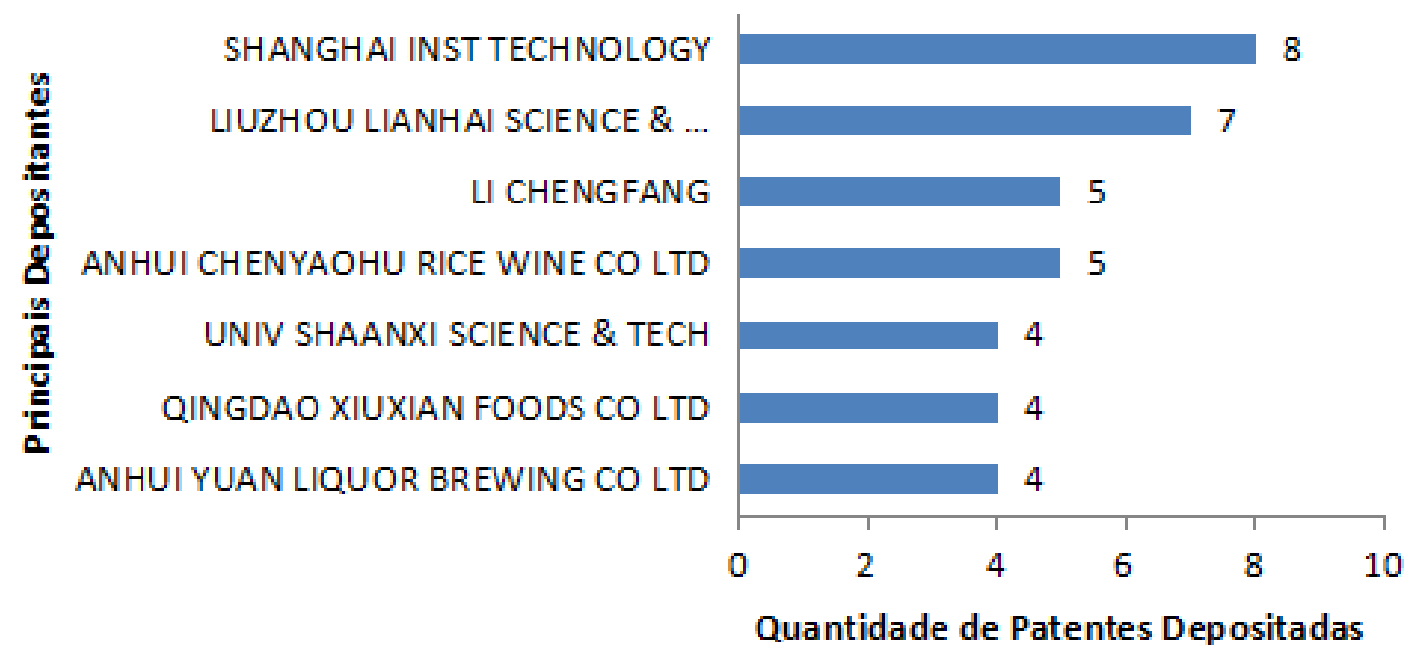

Fonte: Elaborada pelos autores deste artigo a partir das bases de dados INPI (2018) e EPO (2018)

Nesse contexto, observa-se que o nível de inovação e de andamento da economia do país possui relação diretamente proporcional ao número de pedidos de patentes apresentado por instituições de ensino e pesquisa. O investimento em Pesquisa \& Desenvolvimento (P\&D) por empresas de todos os portes e universidades, também, demonstra o grau de produtividade da nação.

Os códigos de Classificação Internacional de maior relevância para o estudo estão apresentados na Figura 6. 
Figura 6 - Distribuição das patentes segundo códigos IPC de maior incidência no estudo

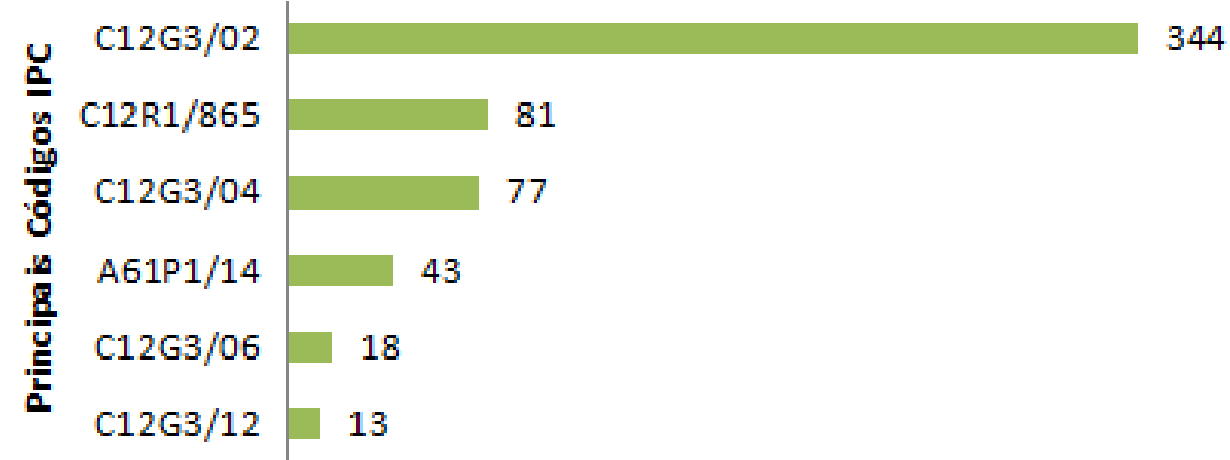

\section{Quantidade de Patentes}

Fonte: Elaborada pelos autores deste artigo a partir das bases de dados INPI (2018) e EPO (2018)

Os códigos IPC C12G3/02, C12R1/865 e C12G3/04 se destacam pelo fato de apresentarem maior incidência no estudo, correspondendo a 39\%, 9\% e 8\% dos códigos presentes nos documentos, respectivamente. Diante disso, pode-se constatar que boa parte das patentes depositadas está relacionada com o interesse do estudo, visto que a pesquisa foi direcionada às classes que englobam as bebidas alcoólicas, entre elas, as fermentadas. Em contrapartida, os códigos A61P1/14, C12G3/06 e C12G3/12 atuaram em 5\%, 2\% e 1\%, respectivamente, nos documentos, revelando que, das inovações na área de bebidas alcoólicas, existe uma porcentagem considerável das bebidas processadas com fins para o apelo funcional e uma porcentagem baixa de bebidas fermentadas que utilizam ingredientes aromatizantes e por destilação no processo. As subdivisões dos códigos e o que consta em cada um estão apresentados na Tabela 4.

Tabela 4 - Códigos com maior incidência e sua abrangência tecnológica

Código IPC

C12G3/02

C12R1/865

C12G3/04

A61P1/14

C12G3/06

$\mathrm{C} 12 \mathrm{G} 3 / 12$

\section{ClassificaÇão}

Por fermentação direta.

Sachharomyces cerevisiae.

Por misturas e licores.

Pró-digestivos, ácidos, enzimas, estimulantes do apetite, antidispépticos, tônicos, antiflatulentos.

Com ingredientes aromatizantes.

Por destilação.

Fonte: EPO (2018)

Sob a ótica de aplicação das diferentes bebidas alcoólicas fermentadas que contêm em sua formulação uma fruta, conforme apresentado na Figura 7, o mirtilo, a amora e o kiwi foram os mais representativos nos documentos de patentes selecionados, estando presente em 38, 31 e 21 documentos de patente, respectivamente; seguidos da ameixa, pera e manga. Além das frutas apresentadas na Figura 7, houve ainda a presença de muitos frutos asiáticos, como: frutas chinesas de magnoliavine, frutas cordyceps sinensis, frutas de arhat, frutos de aronia melanocarpa, frutos de murta, frutos de schisandra, frutos rhodomyrti, frutos de corniso, frutos de índigo, entre outras variedades de espécies cultivadas. 
Figura 7 - Quantitativo de frutas tratadas nos documentos selecionados

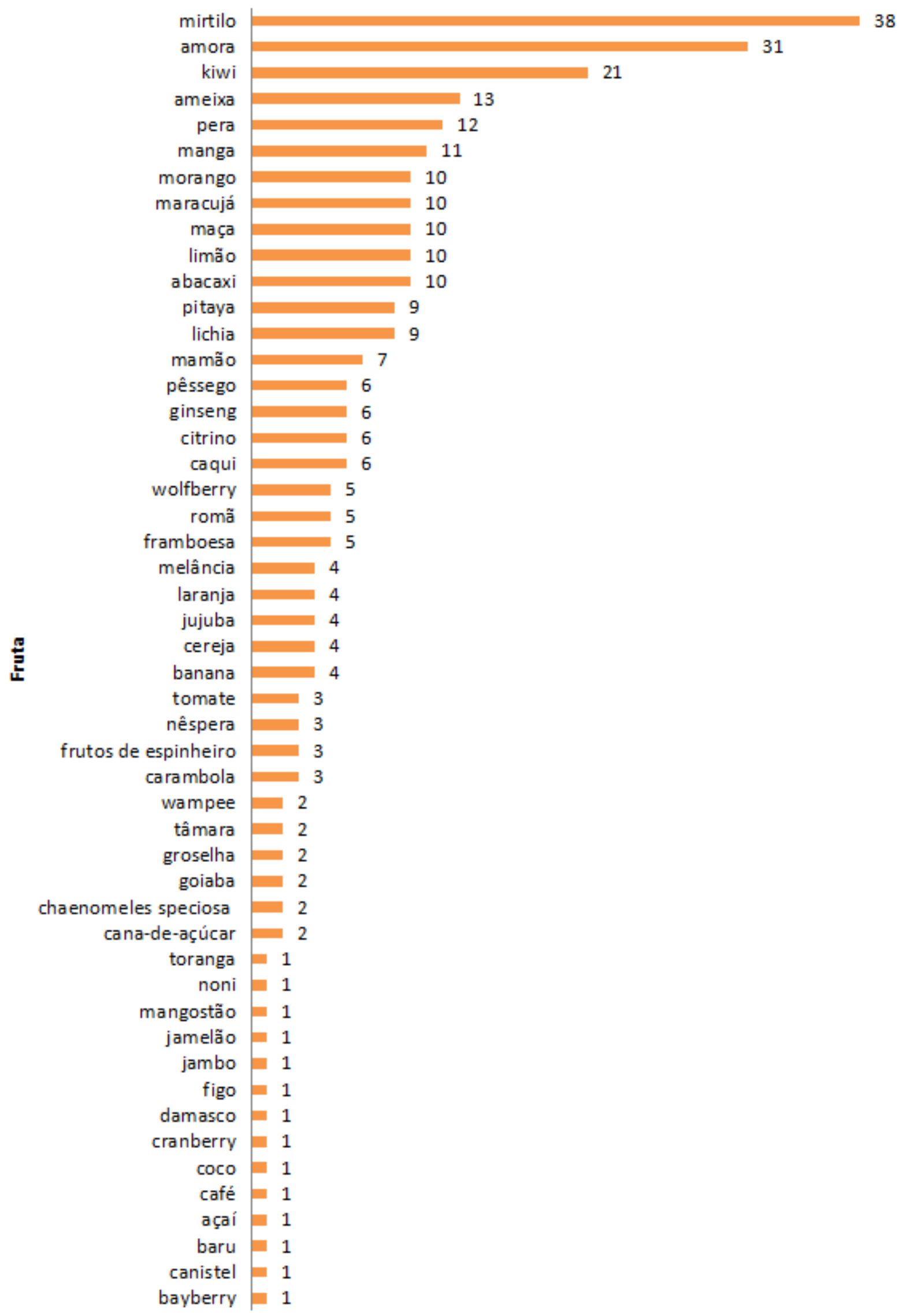

\section{Quantidade de Patentes Depositadas}

Fonte: Elaborada pelos autores deste artigo a partir das bases de dados INPI (2018) e EPO (2018) 


\section{Considerações Finais}

Diante dos dados expostos neste estudo prospectivo, conclui-se que existe uma tendência ao crescimento de depósitos patentárias na área de bebidas e do quanto é importante o incentivo à ciência, à pesquisa e à tecnologia num país, uma vez que o número de solicitações de documentos de patentes depositados pelo país possui relação diretamente proporcional ao desenvolvimento econômico da nação.

Apesar do avanço brasileiro no cenário mundial no depósito de patentes $-11^{a}$ colocação no ranking de depositantes de patentes em 2016 - percebe-se que os incentivos nesse campo ainda são muito escassos, pois o país, que é o terceiro produtor mundial de frutas, foi responsável apenas por cinco patentes depositadas na plataforma INPI.

Paralelo a isso, ressalta-se ainda que a produção de bebidas alcoólicas fermentadas a partir das frutas representa uma forma alternativa para contornar os problemas das perdas pós-colheita, além de auxiliar a inserção de novos produtos no mercado.

\section{Referências}

BRASIL. Ministério da Agricultura, Pecuária e Abastecimento. Portaria n. 64, de 23 de abril de 2008. Regulamento Técnico para a fixação dos padrões de identidade e qualidade para as bebidas alcoólicas fermentadas. Diário Oficial [da] República Federativa do Brasil, Brasília, novembro de 2008. Disponível em: http://www.aladi.org/nsfaladi/normasTecnicas.nsf/09267198f1324b64 032574960062343c/ef1ee2d72487688603257a9f004bbf57/\$FILE/ATTPLES5.pdf/Portaria\%20 N\%C2\%B0\%2064-2008.pdf. Acesso em: 4 jun. 2018.

BRASIL. Ministério da Agricultura, Pecuária e Abastecimento. Instrução Normativa n. 34, de 29 de novembro de 2012. Complementação dos padrões de identidade e qualidade para as bebidas fermentadas. Diário Oficial [da] República Federativa do Brasil, Brasília, novembro de 2012. Disponível em: http://www.agricultura.gov.br/assuntos/vigilancia-agropecuaria/ivegetal/bebidasarquivos/in-no-34-de-29-de-novembro-de-2012.pdf. Acesso em: 4 jun. 2018.

EPO - EUROPEAN PATENT OFFICE. Cooperative Patent Classification. [2018]. Disponível em: https://worldwide.espacenet.com/classification?locale=en_EP. Acesso em: 7 maio 2018.

ROSA S. E. S. da; COSENZA J. P.; LEÃO L. T. de S. Panorama do setor de bebidas no Brasil. BNDS Setorial, Rio de Janeiro, n. 23, p. 101-150, mar. 2006. Disponível em: https://web.bndes. gov.br/bib/jspui/bitstream/1408/2607/1/BS\%2023\%20Panorama\%20do\%20Setor\%20de\%20 Bebidas\%20no\%20Brasil_P.pdf. Acesso em: 6 jun. 2018.

SEBRAE - SERVIÇO BRASILEIRO DE APOIO ÀS MICROS E PEQUENAS EMPRESAS. Análise da Conjuntura Agropecuária - Fruticultura. Safra 2016/2017. [2017]. 9p. Disponível em: http:// www.agricultura.pr.gov.br/arquivos/File/deral/Prognosticos/2017/Fruticultura_2016_17.pdf. Acesso em: 6 jun. 2018.

SEBRAE - SERVIÇO BRASILEIRO DE APOIO ÀS MICROS E PEQUENAS EMPRESAS. Boletim de Inteligência - Agronegócio, Fruticultura, [s.l.], out. 2015. 5p. Disponível em: http://www. bibliotecas.sebrae.com.br/chronus/ARQUIVOS_CHRONUS/bds/bds.nsf/64ab878c176e5103877bfd3f 92a2a68f/\$File/5791.pdf. Acesso em: 6 jun. 2018. 
SEBRAE - SERVIÇO BRASILEIRO DE APOIO ÀS MICROS E PEQUENAS EMPRESAS. Secretaria da Agricultura e do Abastecimento. Departamento de Economia Rural. Boletim de Inteligência Agronegócio, Fruticultura, [s.l.], ago. 2016. 5p. Disponível em: http://www.bibliotecas.sebrae.com. br/chronus/ARQUIVOS_CHRONUS/bds/bds.nsf/3e48870e607bdcc4c945d4a81de6d689/\$File/7265. pdf. Acesso em: 6 jun. 2018.

WIPO - WORLD INTELLECTUAL PROPERTY ORGANIZATION. Economics \& Statistics Series, 2011. Disponível em: http://www.wipo.int/edocs/pubdocs/en/intproperty/941/wipo_pub_941_2011. pdf. Acesso em: 8 jun. 2018.

WIPO - WORLD INTELLECTUAL PROPERTY ORGANIZATION. Economics \& Statistics Series, 2013. Disponível em: http://www.wipo.int/edocs/pubdocs/en/intproperty/941/wipo_pub_941_2013. pdf. Acesso em: 8 jun. 2018.

WIPO - WORLD INTELLECTUAL PROPERTY ORGANIZATION. Economics \& Statistics Series, 2015. Disponível em: http://www.wipo.int/edocs/pubdocs/en/wipo_pub_941_2015.pdf. Acesso em: 8 jun. 2018.

WIPO - WORLD INTELLECTUAL PROPERTY ORGANIZATION. Economics \& Statistics Series, 2017. Disponível em: http://www.wipo.int/edocs/pubdocs/en/wipo_pub_941_2017.pdf. Acesso em: 8 jun. 2018.

\section{Sobre os Autores}

\section{Renata Torres dos Santos e Santos}

E-mail: retorressantos@gmail.com

Mestre em Ciência de Alimentos pela Universidade Federal da Bahia em 2018.

Endereço profissional: Programa de Pós-Graduação em Ciência e Tecnologia de Alimentos, Universidade Federal da Paraíba, Campus I, Centro de Tecnologia, João Pessoa, PB. CEP: 58051-900.

\section{Clívia Danubia Pinho da Costa Castro}

E-mail: clivia.castro@embrapa.br

Doutora em Tecnologia de Alimentos pela Unicamp em 2011.

Endereço profissional: Empresa Brasileira de Pesquisa Agropecuária, Embrapa Semiárido, BR 128, Km 152, Zona Rural, Petrolina, PE. CEP: CEP: 56302-970.

\section{Ana Cecilia Poloni Rybka}

E-mail: ana.rybka@embrapa.br

Doutora em Ciência de Alimentos pela Unicamp em 2010.

Endereço profissional: Empresa Brasileira de Pesquisa Agropecuária, Embrapa Semiárido, BR 128, Km 152, Zona Rural, Petrolina, PE. CEP: CEP: 56302-970.

\section{Aline Camarão Telles Biasoto}

E-mail: aline.biasoto@embrapa.br

Doutora em Alimentos e Nutrição pela Unicamp em 2013.

Endereço profissional: Empresa Brasileira de Pesquisa Agropecuária, Embrapa Semiárido, BR 128, Km 152, Zona

Rural, Petrolina, PE. CEP: CEP: 56302-970. 
Renata Torres dos Santos e Santos, Clívia Danubia Pinho da Costa Castro, Ana Cecilia Poloni Rybka,

Aline Camarão Telles Biasoto, Flávio Luiz Honorato da Silva

\section{Flávio Luiz Honorato da Silva}

E-mail: flavioluizh@yahoo.com.br

Doutor em Engenharia de Alimentos pela Unicamp em 1998.

Endereço profissional: Universidade Federal da Paraíba, Campus I, Centro de Tecnologia, Cidade Universitária, João Pessoa, PB. CEP: 58051-900. 